\title{
Mirtazapine-induced arthralgia
}

\author{
Anneke Passier \& Eugène van Puijenbroek
}

Netherlands Pharmacovigilance Centre Lareb, 's-Hertogenbosch, the Netherlands

\section{Correspondence}

Anneke Passier, PhD, Netherlands Pharmacovigilance Centre Lareb, Goudsbloemvallei 7, $5327 \mathrm{MH}$ 'sHertogenbosch, The Netherlands.

Tel: +31736469718

Fax: +31736426136

E-mail: A.Passier@Lareb.nl

\section{Keywords}

Mirtazapine, arthralgia, adverse drug reaction, serotonin, $5 \mathrm{HT} 1$-receptors

\section{Introduction}

Mirtazapine (Remeron ${ }^{\circledR}$ ) is a tetracyclic piperazinoazepine analogue of mianserin used for treatment of depression. The compound has a chemical structure closely related to mianserin but unrelated to other drugs used in the treatment of depression, like tricyclic antidepressants (TCAs), monoamino oxidase inhibitors (MAOIs), or selective serotonin reuptake inhibitors (SSRIs). Mirtazapine was approved for marketing in the Netherlands in 1994 [1]. Due to its mode of action, mirtazapine can be described as a noradrenergic and specific serotonergic antidepressant (NaSSA) [2].

Although both arthralgia and myalgia are described as possible adverse drug reactions in the product information of mirtazapine, this association has seldom been described in literature. The Netherlands Pharmacovigilance Centre Lareb, maintaining the voluntary adverse drug reaction reporting system in the Netherlands on behalf of the Dutch Medicines Evaluation Board, received eight reports on arthralgia or arthralgia-related symptoms in association with mirtazapine. In this article, we present a short overview of these reports.

\section{Methods and results}

From May 1995 until October 2004, the Netherlands Pharmacovigilance Centre Lareb received eight reports of arthralgia or arthralgia-related symptoms in association with the use of mirtazapine. Details on all eight cases are presented in Table 1.

The time to onset was reported for all patients and was short; varying from 2 to 22 days. Six of the eight 
Table 1

Characteristics of reports of arthralgia and/or arthritis associated with mirtazapine submitted to the Netherlands Pharmacovigilance Centre between May 1995 and October 2004

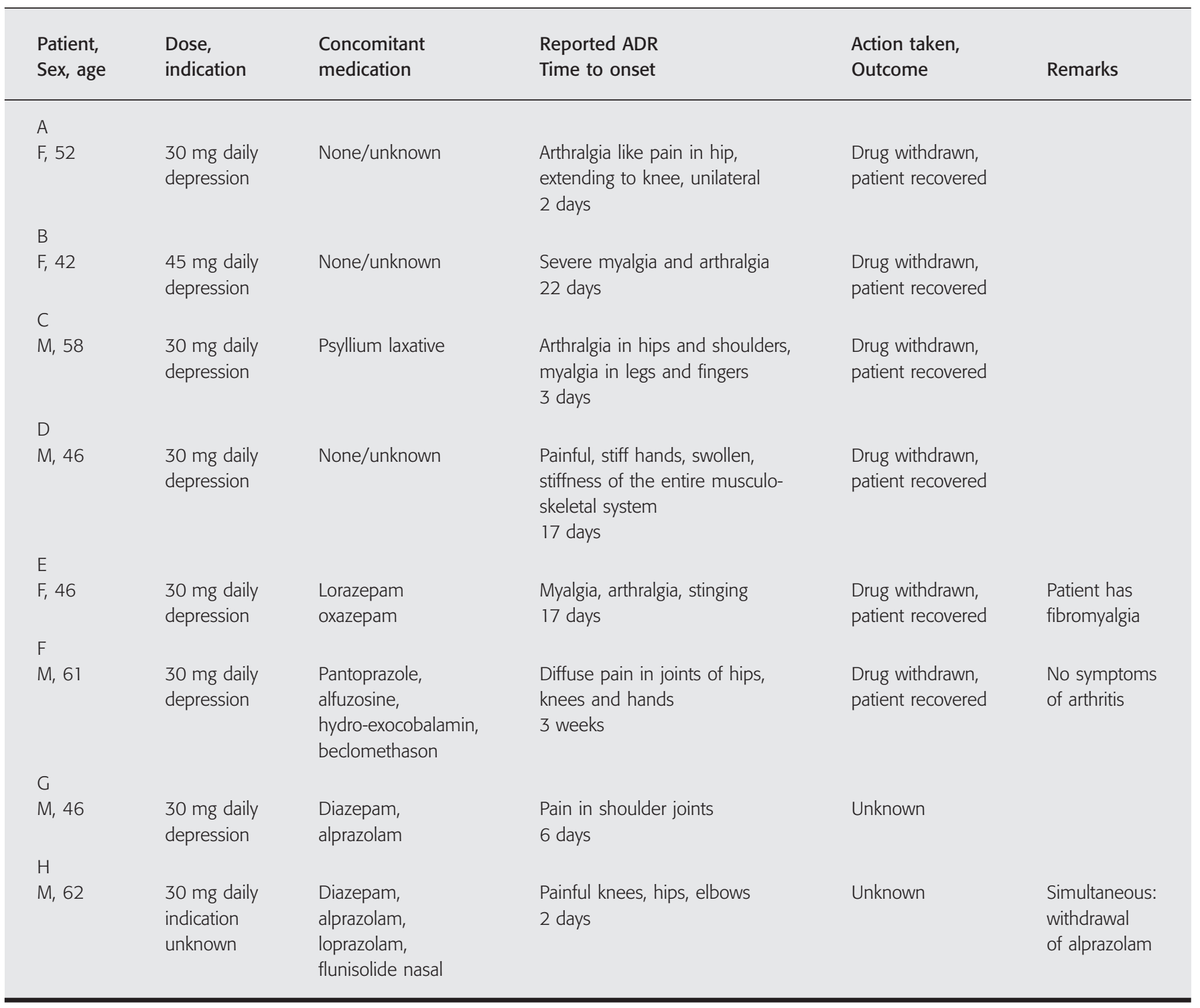

patients discontinued the use of mirtazapine and recovered rapidly. One patient was reported with a disorder in her medical history that may have contributed to the reported symptoms. Nevertheless, the mirtazapineassociated stinging musculoskeletal and joint pain experienced by the 46-year-old female with fibromyalgia (patient $\mathrm{F}$ ) disappeared 2 days after withdrawal of the medication.

Up to March 2004, the worldwide database of the WHO Monitoring Centre contained a total of 4578 reports of adverse drug reactions on mirtazapine. One hundred and ten of these $(2.4 \%)$ concern reports of arthralgia. It is statistically significant that arthralgia is reported in association with mirtazapine more often than expected (odds ratio 1.95; 95\% CI 1.61-2.36).

\section{Discussion}

The association between mirtazapine and arthralgia, demonstrated in both the Lareb and the WHO worldwide databases, is not commonly described in the literature. To our knowledge (Medline search October 2004), Jolliet et al. were the first and only ones to describe two case reports on this association [3, 4]. The reports in this article make a considerable contribution to this number. Arthralgia is also reported in association with other antidepressants, but only for mirtazapine and 
mianserin this association was found to be significant in both the Lareb and the WHO databases (WHO odds ratio on mianserin and arthralgia 3.45 ; $95 \%$ CI $2.97-$ 4.00)*.

The mechanism by which mirtazapine causes this adverse drug reaction is unclear. Mirtazapine has a specific mode of action: it induces enhanced noradrenergic and serotonergic neurotransmission by antagonizing presynaptic $\alpha 2$-receptors. The increase in serotonin synaptic concentrations indirectly enhances 5HT1mediated neurotransmission, since 5HT2 and 5HT3receptors are potently blocked by mirtazapine $[1,2,5]$. This specific effect on the 5HT1-receptors may be a relevant factor in inducing specific adverse drug reactions, including arthralgia.

Jolliet et al. suggested that the structural similarity with mianserin (a tetracyclic antidepressant that is associated with arthralgia more frequently) may be a relevant factor [3]. It should be noted that, besides their striking structural similarity, mianserin and mirtazapine also show similarity with respect to their mechanism of action. Both antidepressants act as central presynaptic $\alpha 2$-adrenergic antagonists and they both antagonize postsynaptic 5HT2-receptors. In line with this hypothesis is the finding that nefazodone, another antidepressant unrelated to TCA, MAOIs or SSRIs, is also associated with arthralgia in the WHO database (odds ratio 1.26; 95\% CI 1.05-1.51) (not significant in the Lareb data- base). Although there is hardly any structural similarity between nefazodone and mirtazapine/mianserin, all three products are known to be 5HT2-antagonists. Hood et al. [6] also suggested that the blockade of 5-HT2areceptors is of importance in induction of arthralgia, based on the mechanism of action of nefazodone.

*The views expressed are purely those of the authors and may not in any circumstances be regarded as stating an official position of WHO.

Competing interests: None declared.

\section{References}

1 The Medicines Evaluation Board. Summary of product characteristics of Remeron ${ }^{\circledR}$ (in Dutch). Version date 2002: http:/ /www.cbg-meb.nl/1B-teksten 16685-16686-18217.pdf

2 De Boer T. The effects of mirtazapine on central noradrenergic and serotonergic neurotransmission. Int Clin Psychopharmacol 1995; 10(Suppl 4): 19-23.

3 Jolliet P, Veyrac G, Bourin M. First report of mirtazapine-induced arthralgia. Eur Psychiatry 2001; 16: 503-5.

4 Veyrac G, Brachet V, Rocher S, Bourin M, Jolliet P. Arthralgia associated with mirtazapine. Therapie 2000; 55 (5): 652-5.

5 Mattia C, Paoletti F, Coluzzi F, Boanelli A. New antidepressants in the treatment of neuropathic pain. A Review. Minerva Anestesiol 2002; 68 (3): 105-14.

6 Hood SD, Argyropoulos SV, Nutt DJ. Arthritis and serotonergic antidepressants. J Clin Psychopharmacol 2001; 21 (4): 458-61. 